\title{
Formation et sentiment d'auto-efficacité des enseignants en compétence informatique et médiatique
}

\section{Stéphanie Boéchat-Heer}

Cet article présente les résultats sur la formation et le sentiment d'auto-efficacité des enseignants en compétence informatique et médiatique issus de l'enquête "International Computer and Information Literacy Study" (ICILS). Cette enquête par questionnaire a été réalisée auprès de 910 enseignants suisses qui exercent leurs activités avec des élèves en $2^{e}$ année $d u$ Cycle secondaire 1 . Les résultats montrent que le sentiment d'auto-efficacité des enseignants en compétence informatique et médiatique est positivement corrélé avec la formation reçue, la fréquence d'utilisation et la collaboration. Ces constats permettent d'apporter des éléments de réflexion et des recommandations pour améliorer les dispositifs de formation dans le domaine.

\section{Introduction}

Les technologies numériques prennent de plus en plus de place dans notre société et se renouvellent en permanence. Les repères, les valeurs, le rapport au savoir, au temps et à l'espace sont modifiés avec l'apparition de différents modes de communication (réseaux sociaux, blogs, etc.). Les "digital natives» (Prensky, 2001) qui vivent avec ces technologies depuis leur naissance, sont très à l'aise avec leur utilisation. Suter et al. (2015) mettent en évidence que la grande majorité des enfants en Suisse a grandi dans un environnement riche en médias et que le comportement d'utilisation évolue et s'intensifie avec l'âge. Les enfants de 6 à 7 ans préferent les livres, les DVD et les vidéos, alors qu'à partir de 10 ou 11 ans, ils utilisent davantage Internet et les téléphones portables. Dans son rapport, l'Organisation de coopération et de développement économiques (OCDE, 2015, p.1), constate que «si les élèves ne sont pas capables de naviguer dans un environnement numérique complexe, ils ne pourront plus participer pleinement à la vie économique, sociale et culturelle du monde qui les entoure». Les enseignants des élèves "connectés» font face à des problématiques complexes, comme l'excès d'informations, le plagiat, la protection des élèves contre les atteintes à la vie privée et le harcèlement en ligne. 
En Suisse, la Confédération et les cantons ont déployé des moyens importants et mis en place des directives pour favoriser l'intégration des Technologies de l'Information et de la Communication (TIC) en classe (Conférence suisse des directeurs cantonaux de l'instruction publique [CDIP], 2007; Conférence intercantonale de l'instruction publique de la Suisse romande et du Tessin (CIIP), 2010). Cependant, les TIC peinent à s'implanter dans les établissements et l'intégration des TIC reste un processus en cours de développement (Boéchat-Heer, 2011; Centre suisse des technologies de l'information dans l'enseignement [CTIE], 2007). L'implantation de dispositifs innovants dans un établissement provoque toujours une certaine réaction des enseignants, leur demande une modification des pratiques existantes et une adaptation. Pour que cette adaptation se passe bien, plusieurs facteurs sont mis en avant par différents travaux: la qualité de la formation; l'accompagnement au sein de l'établissement; la collaboration entre collègues; le soutien des responsables TIC et l'implication de la direction; l'équipement; les projets d'établissement et la culture de l'établissement (Boéchat-Heer, 2012; Fredricksson \& Hoskins, 2007; Niemi, Kynäslahti \& Vahtivuori-Hänninen, 2012; Valiente, 2010). Les enseignants ont besoin d'être coachés, d'être accompagnés dans le processus d'intégration des TIC à leur pratique d'enseignement, que ce soit par des projets d'établissement promus par la direction, ou par la proximité et la disponibilité d'une personne de référence spécialisée dans le domaine. L'accompagnement et le soutien (Valiente, 2010), l'accompagnement et les apprentissages informels dans la résolution de problèmes techniques (Boéchat-Heer, 2012) sont des facteurs facilitant l'intégration. Qu'en est-il des compétences informatiques et médiatiques des enseignants en Suisse? Sont-ils suffisamment formés? Utilisent-ils ces outils dans leur activité quotidienne d'enseignant?

Pour répondre à ces différentes questions, nous nous appuyons sur les données des enseignants suisses issus d'une étude comparative "International Computer and Information Literacy Study» (ICILS). Cette étude a évalué les compétences informatiques et médiatiques des élèves en fin de scolarité obligatoire et des enseignants. Par compétence informatique et médiatique, nous entendons l'habileté individuelle à utiliser les ordinateurs pour chercher, créer et communiquer. Nous avons choisi d'approfondir nos analyses en nous penchant sur les phases d'intégration, la formation et le sentiment d'auto-efficacité des enseignants.

\section{Cadre conceptuel}

\section{Le processus d'intégration des TIC}

Toute innovation implique un processus d'intégration qui se passe en différentes étapes. Plusieurs auteurs se sont penchés sur ces phases et ont proposé un modèle d'intégration des TIC (Mishra \& Koehler, 2006; Moersch, 1995; Morais, 2001; Newby \& Lay, 1996, mars; Sandholtz, Ringstaff \& Dwyer, 
1997). Ces modèles ont l'avantage de définir l'évolution des pratiques pédagogiques lors du processus d'intégration. Cependant, leur principale faiblesse est de soutenir un modèle linéaire dans lequel tous les enseignants traversent les mêmes étapes selon l'ordre proposé. Les enseignants n'ont donc pas la possibilité de passer un niveau ou de régresser. Exceptés les modèles de Morais (2001) et de Newby et Lai (1996, mars), les autres ne font pas la différence entre les usages personnels et les usages professionnels des enseignants. Le modèle de Depover et Strebelle (1997) a l'avantage de proposer une analyse écosystémique, en montrant l'importance de l'environnement sur la pratique des TIC. Il rejoint la position de Piaget sur l'adaptation et l'importance de la relation entre le sujet et l'environnement pour construire les connaissances. Ce modèle est construit selon deux axes: un axe dynamique et un axe topologique. Nous prendrons en considération dans cette étude l'axe dynamique. Ce dernier montre un processus d'innovation en trois temps: adoption; implantation et routinisation. Tout d'abord, la phase d'adoption, dans laquelle les enseignants se motivent et se préparent et qui se caractérise par "la décision de changer quelque chose dans sa pratique par conviction personnelle ou sous une pression externe qui peut s'exercer au départ du microsystème» (Depover \& Strebelle, 1997, p. 80). Puis, la phase d'implantation où les enseignants réalisent des projets d'intégration, des activités d'apprentissage, et ont un sentiment de maîtrise professionnelle. Elle correspond à «la concrétisation sur le terrain de la volonté de s'engager dans un processus conduisant à une modification des pratiques éducatives [...] cette phase se traduit naturellement par des modifications perceptibles au niveau des pratiques éducatives mais aussi de l'environnement dans lequel ces pratiques prennent place» (Depover \& Strebelle, 1997, p. 81). Finalement, les enseignants parviennent à une phase de routinisation qui correspond à une stabilisation, une amplification et une diffusion des activités. Elle se caractérise par le fait que «le recours aux nouvelles pratiques s'opère sur une base régulière et intégrée aux activités scolaires habituelles sans exiger pour cela un support externe de la part d'une équipe de recherche ou d'animation pédagogique» (Depover \& Strebelle, p. 82). Ce modèle insiste sur l'importance de tenir compte du contexte dans lequel s'insère l'innovation. Il donne donc une importance aux aspects systémiques de l'innovation. En nous appuyant sur ce modèle, nous nous attacherons dans un premier temps à définir le niveau d'intégration des enseignants suisses dans le domaine des TIC. Nous mettrons ensuite en relation ce niveau d'intégration avec la formation et le sentiment d'auto-efficacité des enseignants en compétences informatiques et médiatiques.

\section{Formation et sentiment d'auto-efficacité des enseignants à l'usage des TIC}

De nombreux travaux montrent le lien entre le sentiment d'auto-efficacité, l'acceptation de pratiques innovantes et l'adaptation aux changements (Abbit \& Klett, 2007; Boéchat-Heer, 2011; Deaudelin, Dussault \& Brodeur, 2002). Selon Bandura (1997), le sentiment d'efficacité personnelle est un déterminant 
fondamental et immédiat de l'engagement et de la performance du sujet dans la tâche. Les déterminants de l'efficacité personnelle sont donc les résultats de ses expériences antérieures dans l'activité en question, l'observation d'autrui en train d'exécuter la conduite visée, toutes formes de persuasion verbale ou non verbale émanant de son entourage et les indications physiologiques ou d'état émotionnel qui lui parviennent de son organisme (stress, tensions, inquiétudes, ...). Selon Bouffard-Bouchard et Pinard (1988), le sentiment d'efficacité personnelle se définit comme «le jugement que porte une personne sur sa capacité d'organiser et d'utiliser les différentes activités inhérentes à la réalisation d'une tâche à exécuter» (p. 411). Les études réalisées sur le sentiment d'auto-efficacité soulignent l'influence d'un sentiment élevé sur une meilleure adaptation au changement et à l'innovation, sur la réduction de l'anxiété et du stress, ainsi que sur une meilleure acceptation de la formation. Des études se sont d'ailleurs intéressées à l'importance du sentiment d'auto-efficacité et son influence sur la pratique des TIC en classe. Abbit et Klett (2007) et Boéchat-Heer (2011) ont montré de quelle manière la perception de sa propre performance face à l'utilisation de l'ordinateur (self-confidence, self efficacy) a un impact sur la modification des usages et des pratiques d'intégration des TIC. De plus, Deaudelin et al. (2002) ont mis en évidences comment une stratégie de développement professionnel qui favorise le sentiment d'auto-efficacité peut avoir un impact sur l'intégration de l'apprentissage coopératif et sur celui des TIC dans la pratique professionnelle. Deaudelin et al. (2002) promulguent que «les enseignants ayant un fort sentiment d'efficacité personnelle ont une tendance plus grande à l'innovation» (p. 393). Dans le même ordre d'idées, au Japon, Wada (2000) observe que l'usage de l'ordinateur (temps et fréquence) est en relation positive avec le sentiment d'auto-efficacité face à l'ordinateur. Des études montrent l'importance de la relation entre sentiment d'auto-efficacité élevé et attitudes positives à l'égard de nouvelles pratiques enseignantes (Guskey, 1988), ainsi que l'impact positif des cours basés sur les problèmes lors de l'intégration (Abbit \& Klett, 2007) pour favoriser la pratique des TIC en classe. En outre, il est à noter que l'apprentissage coopératif renforce le sentiment d'auto-efficacité des enseignants (TschannenMoran, Woolfolk Hoy \& Hoy, 1998) et qu'il est important de favoriser les expériences de réussite en accompagnant les formés et en donnant des feedbacks positifs (Boéchat-Heer, 2012). L'accompagnement au sein de l'établissement joue aussi un rôle déterminant pour améliorer le sentiment d'auto-efficacité, en favorisant le collaboration entre collègues, le soutien des responsables TIC, l'implication de la direction, l'équipement, les formations et les projets d'établissements, la culture de l'école (Boéchat-Heer, 2012; Fredricksson \& Hoskins, 2007; Niemi, Kynäslahti \& Vahtivuori-Hänninen, 2012; Valiente, 2010). Pajares et Zeldin (1999) montrent que la persuasion verbale et les expériences vicariantes (modelage) constituent des éléments importants dans la construction de l'efficacité personnelle. Dans la présente étude, nous chercherons à évaluer le sentiment d'auto-efficacité des enseignants en TIC et les compétences acquises en formation en lien avec le niveau d'intégration. 


\section{Méthodologie}

Cette recherche quantitative est une enquête par questionnaire qui s'est déroulée de février à avril 2013. Elle s'appuie sur les données des enseignants de Suisse issues de la recherche ICILS. Cette dernière est une étude comparative internationale à laquelle ont participé plus de 20 pays et vise à évaluer les compétences des élèves en fin de scolarité obligatoire et leurs aptitudes à vivre dans la société de l'information en analysant leur capacité à se servir des nouvelles technologies à l'école et en dehors du cadre scolaire. Le questionnaire a été envoyé à 1402 enseignants suisses. Avec un taux de réponses de 64.9\%, l'échantillon est constitué de 910 enseignants répartis dans 99 écoles. Dans chaque école sélectionnée au hasard, 15 enseignants ont été choisis de manière aléatoire parmi les enseignants qui réalisent leurs activités avec des élèves en $2^{\mathrm{e}}$ année du Cycle secondaire 1 ( $2^{\mathrm{e}}$ C.O.; $8^{\mathrm{e}}$; Degré 10). Le questionnaire que nous avons envoyé aux enseignants se compose de différents thèmes: les informations générales; l'expérience et les connaissances en TIC (par exemple: dans quelle mesure pouvez-vous accomplir vous-même les tâches suivantes à l'ordinateur?); l'utilisation des TIC dans l'enseignement avec une classe de $2^{\mathrm{e}}$ année du Cycle secondaire 1 (par exemple: depuis combien de temps utilisez-vous l'ordinateur à des fins d'enseignement?); l'utilisation des TIC à l'école (par exemple: dans quelle mesure êtes-vous d'accord avec l'énoncé suivant: l'utilisation des TIC à l'école permet aux élèves de consulter de meilleures sources d'information?); l'apprentissage de l'utilisation pédagogique des TIC (par exemple: quelle incidence les activités de perfectionnement ont-elles eues sur votre capacité à utiliser les TIC à des fins d'enseignement?). Il comprend 18 questions dont 12 questions ordinales multichotomiques, 5 questions nominales multichotomiques et une question dichotomique. Les enseignants ont été invités à répondre au questionnaire en ligne par courrier électronique. Les données du questionnaire ont été récoltées à l'aide d'une plateforme web qui enregistrait les réponses directement dans la centrale de récolte. Nous avons réalisé en premier lieu une analyse descriptive des données qui va nous permettre de répondre aux questions suivantes: quel est le niveau de formation et quelles sont les compétences informatiques et médiatiques des enseignants? Quel est le sentiment d'auto-efficacité des enseignants dans ce domaine? Quel est le niveau d'intégration des TIC par les enseignants? Dans un deuxième temps, par une méthode corrélationnelle et une analyse inférentielle à l'aide du logiciel SPSS, nous avons mis en relation les différentes variables utilisées (niveau d'intégration, formation, sentiment d'auto-efficacité, collaboration) pour mieux comprendre les résultats. Dans quelle mesure le niveau de formation des enseignants en TIC est en relation avec leur sentiment d'auto-efficacité dans le domaine? Dans quelle mesure le sentiment d'auto-efficacité en TIC des enseignants est en relation avec la fréquence d'utilisation en classe? Dans quelle mesure le sentiment d'auto-efficacité des enseignants est en relation avec la collaboration? 


\section{Résultats}

\section{Niveau d'intégration des TIC dans les écoles}

Nous commençons l'analyse des résultats par la présentation des caractéristiques générales des enseignants qui ont répondu au questionnaire. Les enseignants sont en majorité des femmes (53\%) avec un âge médian de 44 ans. Les enseignants sont représentés par discipline en majorité en langue première; langues étrangères et langues nationales; mathématiques. La majorité des enseignants (87\%) utilisent les TIC à des fins personnelles depuis deux ans ou plus. Seuls 5\% n'ont jamais utilisé les TIC et $8 \%$ utilisent les TIC depuis moins de deux ans.

En ce qui concerne le niveau d'intégration des enseignants, le le graphe 1 ci-dessous ci-dessous montre que $76 \%$ des enseignants utilisent quotidiennement les TIC en dehors de l'école, $58 \%$ des enseignants les utilisent à l'école pour d'autres tâches liées au travail et $27 \%$ les utilisent quand ils enseignent. Nous remarquons que plus de la moitié des enseignants les utilisent au moins une fois par semaine (53\%) à l'école quand ils enseignent.

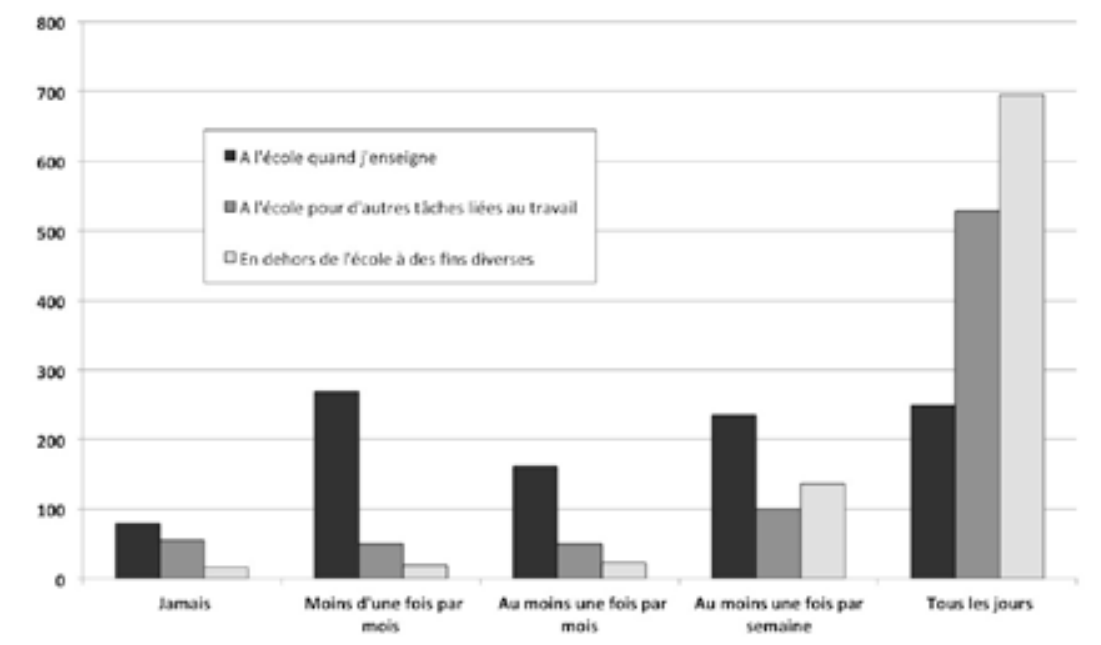

Graphe 1: la fréquence d'utilisation des TIC par l'enseignant selon le type d'activités

Nous continuons la présentation des résultats en nous penchant plus particulièrement sur le type de pratiques et leur fréquence d'utilisation. Sur une échelle allant de «jamais» à "pour chaque leçon», les résultats montrent que les outils TIC les plus utilisés pour quelques leçons en classe sont les ressources informationnelles sur ordinateur (par ex. Wikipédia, sites internet) (42\%); les logiciels de traitement de texte ou de présentation (36\%); les tutoriels ou logiciels d'exercice $(36 \%)$. Les enseignants n'utilisent presque jamais le portfolio électronique (97\%), les logiciels de schématisation conceptuelle (95\%), les réseaux sociaux (Facebook, twitter) (94\%). Au niveau des types d'activités réalisées en classe avec 
les TIC, les résultats indiquent que les enseignants réalisent avec leurs élèves un peu plus souvent des activités de courte durée (parfois $=38 \%$ et souvent $=10 \%$ ) que de longue durée (parfois $=30 \%$ et souvent $=9 \%$ ). Plus d'un quart des enseignants disent réaliser parfois les activités suivantes avec leurs élèves: travailler individuellement avec un matériel pédagogique, à leur propre rythme (32\%); suivre une démarche d'investigation ou faire une recherche sur le terrain sans limite dans le temps (33\%); chercher de l'information sur un sujet à l'aide de ressources extérieures (37\%). Ces différentes activités témoignent d'une utilisation des TIC comme appui à l'enseignement traditionnel, que ce soit par la recherche d'informations sur Internet pour approfondir un sujet ou que ce soit pour renforcer certaines notions par un travail individuel et différencié au travers des activités de drill. Selon le modèle de Depover et Strebelle (1997), la majorité des enseignants se situent en phase d'adoption, parce qu'ils se servent des TIC comme appui à leur enseignement. Il peut s'agir d'occuper un élève qui a de l'avance ou aider un élève qui a plus de difficultés (didacticiels), de rechercher de l'information sur internet. Les enseignants se motivent et se préparent. Nous pouvons difficilement affirmer que les enseignants réalisent fréquemment des projets d'intégration avec leurs élèves, des activités d'apprentissage, et ont un sentiment de maitrise professionnelle. Il ressort que plus des deux tiers des enseignants n'utilisent presque jamais les types d'activités suivantes dans leur classe: évaluer les apprentissages des élèves au moyen de tests (73\%); fournir une rétroaction aux élèves (75\%); favoriser la collaboration entre les élèves $(74 \%)$; faciliter la communication entre les élèves et des spécialistes ou des mentors de l'extérieur (91\%); aider les élèves à collaborer avec d'autres (de la même école ou d'autres écoles) (83\%); collaborer avec les parents ou les tuteurs pour favoriser l'apprentissage chez les élèves (84\%). Il est à noter également que plus d'un quart des enseignants utilisent parfois les activités suivantes: présenter de l'information au moyen de consignes directes à la classe (34\%); offrir des classes d'appoint ou d'enrichissement à des élèves, individuellement ou en petits groupes (30\%); faire en sorte que les élèves puissent discuter ou faire des présentations à toute la classe (30\%); consolider les apprentissages par la répétition d'exemples (29\%) et faciliter l'apprentissage par la découverte (34\%). Nous retrouvons à travers ces résultats que les enseignants utilisent les TIC comme appui à leur enseignement, pour des activités de présentation (ppt) ou pour des activités d'appui à certains élèves en difficulté (répétition d'exercices). Les enseignants n'utilisent que rarement les TIC comme outils de communication et d'évaluation des apprentissages. Nous pouvons ainsi conclure que la majorité des enseignants suisses ne se situent pas encore en phase d'implémentation ou de routinisation. Les enseignants qui se situent en phase d'implémentation s'engagent «dans un processus conduisant à une modification des pratiques éducatives [...] cette phase se traduit naturellement par des modifications perceptibles au niveau des pratiques éducatives mais aussi de l'environnement dans lequel ces pratiques prennent place» (Depover \& Strebelle, 1997, p. 81). En phase de routinisation, «le recours 
aux nouvelles pratiques s'opère sur une base régulière et intégrée aux activités scolaires habituelles sans exiger pour cela un support externe de la part d'une équipe de recherche ou d'animation pédagogique» (Depover \& Strebelle, 1997, p. 82). À partir de ce constat, il importe d'évaluer si le fait que les enseignants soient en phase d'adoption soit en lien avec la formation des enseignants en TIC et le sentiment d'auto-efficacité.

\section{Formation et sentiment d'auto-efficacité en TIC}

Nous nous penchons tout particulièrement sur la formation des enseignants en TIC et le sentiment d'auto-efficacité dans le domaine. Les résultats montrent tout d'abord que les enseignants sont formés à des compétences très différentes. Environ un tiers des enseignants (31.4\%) sont formés à des compétences pédagogiques et didactiques en TIC (cours sur l'intégration des TIC à l'enseignement et l'apprentissage, formation à l'usage d'un logiciel dédié à une matière précise, observations des professeurs, etc.). Les autres enseignants sont représentés dans différents domaines de formation comme l'utilisation d'outils spécifiques. Moins d'un quart des enseignants ont reçu des cours d'initiation à des applications générales (par ex., traitement de textes, tableurs et bases de données de base) (19.5\%), des cours supérieurs sur des applications générales (p. ex., traitement de textes, tableurs et bases de données avancés) (12.7\%), des cours d'initiation à l'usage d'Internet (p. ex., compilation de recherches sur Internet, ressources numériques) (16.6\%), des cours supérieurs sur l'usage d'Internet (p. ex., création d'un site ou de ressources Internet) (10.7\%).

Nous présentons maintenant les résultats liés au sentiment d'auto-efficacité des enseignants. Sur une échelle à trois degré (je sais comment le faire, je pourrais arriver à le faire, je ne pense pas pouvoir le faire), nous constatons que les enseignants se sentent davantage compétents (je sais comment le faire) dans l'utilisation des outils TIC suivants: rédiger une lettre à l'aide d'un logiciel de traitement de textes $(97 \%)$; envoyer un fichier par courriel en pièce jointe $(97 \%)$; classer des documents numériques (94\%); trouver des ressources pédagogiques utiles sur Internet (93\%); mettre en mémoire vos photos numériques sur un ordinateur (91\%); utiliser Internet pour faire des achats et des paiements en ligne (92\%). Ils se sentent moyennement compétents dans les tâches liées à l'enseignement avec les TIC: surveiller les progrès des élèves (49\%); évaluer les apprentissages des élèves (51\%). Les compétences liées à l'utilisation d'outils sont souvent acquises par auto-formation dans le but d'une utilisation personnelle. Ces résultats sont en lien direct avec les constats liés à la formation en TIC qui montrent que les enseignants sont davantage formés à des compétences techniques plutôt qu'à des compétences pédagogiques et didactiques.

Un autre constat intéressant montre que l'échange et la collaboration entre les enseignants sur la pratique des TIC en classe est relativement faible. Environ la 
moitié des enseignants disent ne jamais discuter avec des collègues des objectifs des leçons (56\%), des méthodes pédagogiques (58\%), ne jamais travailler avec d'autres collègues à l'élaboration des leçons utilisant les TIC (42\%), ne jamais observer d'autres collègues utiliser les TIC à des fins d'enseignement (42\%). Ces résultats rejoignent les constats sur l'importance de l'accompagnement au sein de l'établissement pour améliorer le sentiment d'auto-efficacité, en favorisant le collaboration entre collègues, le soutien des responsables TIC, l'implication de la direction, l'équipement, les formations et les projets d'établissements (Boéchat-Heer, 2012; Fredricksson \& Hoskins, 2007; Niemi, Kynäslahti \& Vahtivuori-Hänninen, 2012; Valiente, 2010). Deux déterminants de l'efficacité personnelle proposés par Bandura (1997) sont liés à l'aspect collaboratif et à l'environnement: «l'observation d'autrui en train d'exécuter la conduite visée» et "toutes formes de persuasion verbale ou non verbale émanant de son entourage» (Bandura, 1997). Ces aspects sont en lien direct avec le sentiment d'auto-efficacité et demandent à être renforcés, tout comme les cours de formation basées sur les compétences pédagogiques et didactiques. Les enseignants suisses ont été formés à des compétences techniques, - l'utilisation d'outils spécifiques -, qui évoluent rapidement et demandent une auto-formation permanente. Ils se sentent compétents par rapport à l'outil pour lequel ils ont été formés mais se sentent moins compétents lorsqu'il s'agit d'enseigner avec les TIC en classe. Pour comprendre davantage les résultats descriptifs que nous avons obtenus, il nous semble important de nous interroger sur les relations entre la formation reçue, le sentiment d'auto-efficacité en TIC, la fréquence d'utilisation et la collaboration.

\section{Relation entre formation, sentiment d'auto- efficacité en TIC, fréquence d'utilisation et collaboration}

Nous observons une corrélation positive et significative $(r=0.181, \mathrm{p}<0,001)$ entre le sentiment d'auto-efficacité et la formation. Ainsi, plus les enseignants sont formés en TIC, plus ils se sentent compétents dans le domaine. Par exemple, s'ils ont reçu un cours sur l'intégration des TIC à l'enseignement et à l'apprentissage, ils se sentiront plus compétents pour préparer des leçons impliquant l'utilisation des TIC avec leurs élèves. Ce constat corrobore les résultats de Deaudelin, Dussault et Brodeur (2002) qui mettent en évidences comment une stratégie de développement professionnel favorisant le sentiment d'auto-efficacité peut avoir un impact sur l'intégration de l'apprentissage coopératif et sur celui des TIC dans la pratique professionnelle. Nous observons également une corrélation positive et significative entre le sentiment d'auto-efficacité et la fréquence d'utilisation des TIC $(r=0,322, p<0,001)$. Plus les enseignants se sentent compétents en TIC, plus ils utilisent les TIC en classe. Ce résultat rejoint les constats de Abitt et Klett (2007) et Boéchat-Heer (2011), qui montre un lien 
entre la perception de sa propre performance face à l'utilisation de l'ordinateur (self-confidence, self efficacy) et la modification des usages et des pratiques d'intégration des TICE et de Wada (2000) qui observe que l'usage de l'ordinateur (temps et fréquence) est en relation positive avec le sentiment d'auto-efficacité face à l'ordinateur. Finalement, nous notons une corrélation positive et significative $(r=0,153, p<0,001)$ entre le sentiment d'auto-efficacité et la collaboration. Ainsi, les enseignants qui se sentent les plus compétents sont ceux qui collaborent le plus. Comme le montrent Tschannen-Moran, Woolfolk Hoy et Hoy (1998), l'apprentissage coopératif renforce le sentiment d'auto-efficacité des enseignants. Ainsi l'accompagnement au sein de l'établissement en favorisant la collaboration entre collègues, le soutien des responsables TIC, l'implication de la direction, les projets d'établissements (Boéchat-Heer, 2012; Fredricksson \& Hoskins, 2007; Niemi, Kynäslahti \& Vahtivuori-Hänninen, 2012; Valiente, 2010) est un élément important permettant de renforcer le sentiment d'auto-efficacité et la pratique future des TIC en classe.

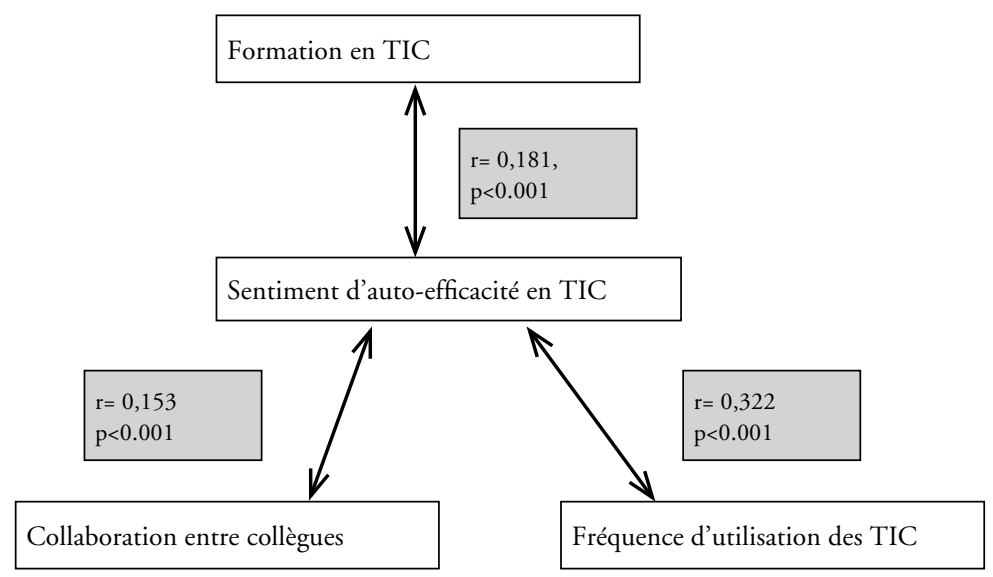

Graphe 2: Relation entre formation, sentiment d'auto-efficactié, fréquence d'utilisation et collaboration entre collègues

\section{Discussion}

En comparaison internationale, la Suisse se situe proche ou en dessus de la moyenne des pays ICILS au niveau de l'équipement et des ressources techniques et en dessous de la moyenne (en fin de classement) en ce qui concerne l'utilisation des TIC en classe pour l'enseignement et l'apprentissage avec leurs élèves. Dans le processus d'intégration des TIC, les résultats montrent que les enseignants se situent en phase d'adoption des TIC selon le modèle de Depover et Strebelle (1997). Ils ont été formés à des compétences très hétérogènes liées à des outils 
spécifiques, mais peu d'enseignants ont eu l'occasion d'être formés à des compétences pédagogiques et didactiques en TIC. Nous observons une corrélation positive et significative $(r=0.181, \mathrm{p}<0,001)$ entre le sentiment d'auto-efficacité et la formation. Ainsi, plus les enseignants sont formés en TIC, plus ils se sentent compétents dans le domaine. Nous observons bien que les enseignants utilisent les TIC dans le domaine pour lequel ils ont été formés. Il est donc important en formation initiale et continue des enseignants de proposer des cours de formation aux compétences pédagogiques et didactiques en TIC. Dans le cadre de chaque discipline, un exemple de pratique qui apporte une plus value pédagogique, devrait être proposé aux étudiants en formation. Tout étudiant devrait également avoir l'occasion de s'exercer en stage et d'être accompagné dans ce processus d'intégration par des feedbacks positifs. Il serait intéressant dans une future recherche de se pencher sur les programmes de formation en TIC dans les Hautes écoles en Suisse, de mesurer le lien entre programmes de formation et usages dans la pratique en classe. Est-ce qu'une formation davantage axée sur les compétences pédagogiques et didactiques en TIC améliorent sensiblement le niveau d'intégration des TIC dans les classes au niveau Suisse?

Les résulats montrent une corrélation positive et significative $(r=0,153$, $\mathrm{p}<0,001)$ entre le sentiment d'auto-efficacité et la collaboration. Ainsi, les enseignants qui se sentent les plus compétents en TIC sont ceux qui collaborent le plus. Comme le montrent Tschannen-Moran, Woolfolk Hoy et Hoy (1998), l'apprentissage coopératif renforce le sentiment d'auto-efficacité des enseignants. Dans une recherche future, il serait également important d'observer l'effet de l'établissement (la collaboration entre collègues, le soutien des responsables TIC, l'implication de la direction, les projets d'établissements) sur le niveau d'intégration des TIC. Est-ce qu'un établissement qui met en place un tel projet aurait un meilleur niveau d'intégration?

Les limites de la recherche réside dans le traitement des valeurs manquantes qui ont réduit sensiblement la taille de notre échantillon et ainsi les effets statistiques de certaines analyses. De plus, l'évaluation du sentiment d'auto-efficacité reposant essentiellement sur différents items du questionnaire permet d'obtenir une vision générale mais rend difficile la mesure d'une note. Un questionnaire spécialisé dans ce type de mesure en complément aurait permis de parvenir à un degré plus important d'objectivité. 


\section{Références}

Abbit, J. T. \& Klett, M. D. (2007). Identifying influences on attitudes and self-efficacy beliefs towards technology integration among pre-service educators. Electronic Journal for the Integration of Technology in Education, 6.

Bandura, A. (1997). Self-efficacy: the exercise of control. New York, NY: W.H. Freeman and Company.

Boéchat-Heer, S. (2011). L'adaptation des enseignants aux usages des MITIC: sentiment d'auto-efficacité, formation et pratiques en classe. Sarrebruck, Allemagne: Editions Universitaires Européennes.

Boéchat-Heer, S. (2012). Evaluation d'une formation aux TICE: développement de compétences et sentiment d'auto-efficacité. In S. Boéchat-Heer \& B. Wentzel (Éd.), Génération connectée: quels enjeux pour l'école? (pp. 151-166). Bienne, Suisse: Editions BEJUNE.

Bouffard-Bouchard, T. \& Pinard, A. (1988). Sentiment d'auto-efficacité et exercice des processus d'auto-régulation chez des étudiants de niveau collégial. International Journal of Psychology, 23, 409-431.

CDIP. (2007). Stratégies de la CDIP en matière de technologies de l'information et de la communication (TIC) et de médias. Berne, Suisse: CDIP.

Conférence intercantonale de l'instruction publique de la Suisse romande et du Tessin (CIIP). (2010). Plan d'études romand (PER). Neuchâtel, Suisse: CIIP.

CTIE. (2007). L'ordinateur et Internet dans les écoles suisses: état et développement entre 2001 et 2007. Berne, Suisse: CTIE.

Deaudelin, C., Dussault, M. \& Brodeur, M. (2002). Impact d'une stratégie d'intégration des TIC sur le sentiment d'auto-efficacité d'enseignants du primaire et leur processus d'adoption d'une innovation. Revue des sciences de l'éducation, 28, (2), 391-410.

Depover, C. \& Strebelle, A. (1997). Fondements d'un modèle d'intégration des activités liées aux nouvelles technologies de l'information dans les pratiques éducatives. In G.-L. Baron \& E. Bruillard (Éd.), Informatique et éducation: regards cognitifs, pédagogiques et sociaux (pp. 9-20). Paris, France: INRP.

Fredriksson, U. \& Hoskins, B. (2007). The development of learning to learn in a European context. Curriculum Journal, 18, (2), 127-134 .

Guskey, T. R. (1988). Teacher efficacy, self concept, and attitudes towards the implementation of instructional innovation. Teaching and Teacher Education, 4, (1), 63-69.

Mishra, P. \& Koehler, M. J. (2006). Technological pedagogical content knowledge: A framework for integrating technology in teachers' knowledge. Teachers College Record, 108, (6), 1017-1054.

Moersch, C. (1995). Levels of technology implementation (LoTi): A framework for measuring classroom technology use. Learning and leading with Technology, 23, (3), 40-42.

Morais, M. A. (2001). Les 5 niveaux d'appropriation des technologies de l'information et de la communication chez les enseignantes et les enseignants. Manuscript non publié, Shédiacs, N.-B., District scolaire ${ }^{\circ} 1$.

Newby, N T. J. \& Lay, F. Q. (1996, mars). Reaching for the next level of computer integration expertise. Papier presenté au Annual meeting of the Society for Informatic Technology and Teacher Education, Phoenix, Arizona.

Niemi, H., Kynäslahti, H. \& Vahtivuori-Hänninen, S. (2012). Towards ICT in everyday life in Finnish schools: Seeking conditions for good practices. Learning, Media and Technology, $1,1-15$.

OCDE. (2015). Connectés pour apprendre: les élèves et les nouvelles technologies. Paris, France: OCDE.

Pajares, F. \& Zeldin, A. L. (1999). Inviting self-efficacy revisited: the role of invitations in the lives of women with mathematics-related careers. Journal of Invitational Theory and Practice, 6, 48-68. 
Prensky, M. (2001). Digital Natives, Digital Immigrants Part 1. On the Horizon, 9, (5), 1-6.

Sandholz, J. H., Ringsta , C. \& Dwyer, D. C. (1997). Teaching with technology: Creating student centered classrooms. New York, NY: Teachers College Press.

Suter, L., Waller, G., Genner, S. Oppliger, S., Willemse, I., Schwarz, B. \& Süss, D. (2015). MIKE: Medien, Interaktion, Kinder, Eltern. Zürich, Suisse: ZHAW.

Tschannen-Moran, M., Woolfolk Hoy, A. \& Hoy, W.K. (1998). Teacher efficacy: its meaning and measure. Review of Educational Research, 68, 202-248.

Valiente, O. (2010). 1-1 in Education. Current Practice, International Comparative Research Evidence and Policy Implications (OECD Education Working Papers, 44). Paris, France: OECD Publishing.

Wada, M. (2000). The empirical study of computer self-efficacy and self-efficacy in computer use. Tokyo Gakugei Daigaku Educational Technology research and Development, 24, 43-53.

Mots-clés: Formation des enseignants, sentiment d'auto-efficacité, compétence informatique et médiatique

\section{Ausbildung und Lehrerselbstwirksamkeit im Zusammenhang mit Computer-und Informationskompetenz}

\section{Zusammenfassung}

Dieser Artikel stellt die Ergebnisse zur Ausbildung und zur Lehrer-Selbstwirksamkeit hinsichtlich ihrer Computer-und Informationskompetenz vor, die von der internationalen Untersuchung ICILS stammen. Diese Fragebogenuntersuchung ist bei 910 Schweizer Lehrpersonen realisiert worden, die Schülerinnen und Schüler der zweiten Klasse auf Sekundarstufe I unterrichten. Die Ergebnisse zeigen eine positive Korrelation zwischen der Lehrer-Selbstwirksamkeit in Computer-und Informationskompetenz und Bildung, der Benutzungshäufigkeit und der Zusammenarbeit. Diese Befunde liefern eine Grundlage zur Reflexion und ermöglichen Empfehlungen zur Verbesserung der Ausbildung in diesem Bereich.

Schlagworte: Lehrerausbildung, Gefühl der Selbstwirksamkeit, Computer- und Informationskompetenz 


\section{Formazione degli insegnanti e senso di autoefficacia nelle competenze informatiche e relative ai media}

\section{Riassunto}

Questo articolo presenta dati relativi alla formazione degli insegnanti e al loro senso di autoefficacia in competenze informatiche e relative ai media derivanti dalla ricerca "International Computer and Information Literacy Study" (ICILS). Questo sondaggio è stato condotto su 910 insegnanti svizzeri che lavorano con gli alunni nel secondo anno del ciclo secondario 1 . I risultati mostrano che il senso di autoefficacia degli insegnanti nelle competenze informatiche e relative media è correlato positivamente con la formazione ricevuta, la frequenza di utilizzo e la collaborazione. Queste osservazioni ci consentono di fornire elementi di riflessione e raccomandazioni per migliorare i dispositivi di formazione sul campo.

Parole chiave: Formazione degli insegnanti, senso di autoefficacia, competenze informatiche e media

\section{Training and teachers' sense of self-efficacy in computer and information literacy}

\section{Summary}

This paper presents the results concerning teacher training and teachers' sense of self-efficacy in computer and information literacy, from the international survey ICILS. This questionnaire survey was carried out among 910 Swiss secondary school teachers. The results show that the teachers' sense of self-efficacy in computer and information literacy is positively related to the training received, the frequency of the use and collaboration. These findings provide elements for reflection and ways to improve training devices in the field.

Keywords: Teacher training, sense of self-efficacy, computer and information literacy 